\title{
Cáncer de próstata de alto riesgo y diseminado (Documento de Consenso de Recomendaciones)
}

\author{
Grupo de Expertos en Cáncer de Próstata
}

Coordinadores: Dr. David Castro Díaz, Vocal de Actividades Científicas, AEU. Dr. Antoni Gelabert Mas, Coordinador Nacional de Urología Oncológica, AEU.

\begin{abstract}
Ponentes: Dr. Carlos Allepuz Llosa, Dr. Joaquín Carballido Rodríguez, Dr. José Manuel Cozar Olmo, Dr. Francisco Gómez-Veiga, Dr. Carlos Hernández Fernández, Dr. Carlos Llorente Abarca, Dr. Juan Morote Robles, Dr. Alfredo Rodríguez-Antolín, Dr. Eduardo Solsona Narbón, Dr. Ángel Tabernero Prieto, Dr. Miguel Unda Urzaiz, Dr. Humberto Villavicencio Mavrich
\end{abstract}

Actas Urol Esp 2005; 29 (9): 826-827

\section{CÁNCER DE PRÓSTATA DE ALTO RIESGO}

Definición

Son varias las definiciones que se pueden encontrar en la literatura, pero coinciden en el mal pronóstico del mismo; como más aceptadas tenemos:

- pacientes con un riesgo mayor del 50\% de presentar recidiva bioquímica en 5 años después de un tratamiento con intencionalidad curativa y riesgo mayor de fallecer por causa cáncer-específica

- pacientes con: $\mathrm{PSA}>\mathrm{o}=20$

$$
\mathrm{y} / \mathrm{o}
$$

Gleason: 8-10

$\mathrm{y} / \mathrm{o}$

$$
\mathrm{cT}>\mathrm{o}=\mathrm{cT} 2 \mathrm{c}
$$

Los tres parámetros son los más utilizados, pero se asume que dos de ellos son suficientes.

Es posible incluir otras variables...?. A título de recomendación incluir la información de las biopsias y el \% de cilindros positivos.

Se recomienda $\mathrm{AL}$ MENOS la biopsia por octantes $(4 \times 4)$ ya que la de sextantes es insuficiente. Se debería tener un patólogo de referencia y es recomendable enviar los cilindros orientados. La información de los cilindros puede orientar sobre la extensión extraprostática.

\section{Tratamiento}

a) El BAM (bloqueo androgéncio máximo) en cualquier modalidad solamente proporciona beneficios modestos comparados con castración (química o quirúrgica), por lo que con las evidencias actuales su uso en estos pacientes de alto riesgo no está recomendado. b) La cirugía radical presenta un elevado riesgo de márgenes afectados y de extensión extraprostática, por lo que en un muy elevado número de pacientes es requerida terapia complementaria. No está recomendada como monoterapia en estos pacientes.

La radioterapia externa tiene que ser de alta dosis ya que es la única efectiva en el Ca. de Próstata de alto riesgo, siendo conscientes de la mayor incidencia de complicaciones; la dosis ideal oscila alrededor de los 75 Gy administrados necesariamente con tecnología adecuada: radioterapia conformacional de intensidad modulada.

La Braquiterapia, aún alcanzando altas dosis, utilizada aisladamente presenta alta tasa de recidivas, por lo que se recomienda sea utilizada como terapia complementaria, no como monoterapia inicial.

La monoterapia no es recomendable como tratamiento de intencionalidad curativa en el Ca. de Próstata de alto riesgo y por lo tanto hay que asociar siempre tratamientos complementarios, por lo que se aconsejan combinaciones de:

- Cirugía + Radiot. Externa

- Cirugía + Braquiterapia

- Radiote. Externa + Hormonoterapia

Cualquiera de estas modalidades tiene sus ventajas e inconvenientes.

\section{Recidiva}

Se define la recidiva bioquímica como el tiempo medio del nadir-primer incremento de PSA. Se sabe que el tiempo que tarda en alcanzar el nadir 
post-radioterapia varía entre 6 y 36 meses y que hay un efecto rebote postradioterapia entre el 12$33 \%$ de pacientes.

En relación a la recidiva bioquímica post Radioterapia y/o Braquiterapia, aconsejamos seguir los criterios de ASTRO para identificarla: tres incrementos sucesivos de PSA, independientemente de su valor, ello siempre sujeto a mejoría o cambios en relación a nuevos estudios.

Se aconseja efectuar determinaciones de PSA cada 3-4 meses durante los dos primeros años y cada 6 meses posteriormente, luego de la aplicación de la radioterapia.

Terapia de rescate post-Radioterapia

Se deben dar unas condiciones mínimas en los pacientes:

- expectación de vida mayor de 10 años

- clínicamente localizado pre y post-radioterapia

- PSA pre-radioterapia y pre-rescate $<10 \mathrm{ng} / \mathrm{ml}$

- Gleason < 6-7

- biopsia pre-rescate (+)

- paciente motivado e informado

- tiempo de doblaje de PSA $>6$ meses

- tiempo hasta la recidiva $>1$ año

No existe evidencia de que la biopsia antes de 2 años aporte ventajas pronósticas; a partir de $\operatorname{los} 2$ años no hay evidencia pronóstica, pero puede ser útil para decidir opciones terapéuticas:

- cirugía radical

- crioterapia

- braquiterapia

- Hifu

\section{CÁNCER DE PRÓSTATA METASTÁSICO}

Tratamiento

El tratamiento hormonal sigue siendo la base terapéutica estándar; en los pacientes del grupo de buen pronóstico con el BAM no existen ventajas a 1-2 años y el coste efectividad es mucho más alto; en el tratamiento con BAM existe un incremento importante de los efectos secundarios, por lo que (y siguiendo a la AEU en orientaciones del año 2003) el BAM no es recomendado más allá de las 4-6 semanas de inicio, para proseguir con análogos solos.

En relación a la monoterapia con antiandrógenos, separados por grupos, no hay aumento de la superviviencia y en los de buen pronóstico puede aumentar la mortalidad. Las reacciones adversas son peor toleradas: ginecomastia y mastodinia.

Los pacientes con cáncer de próstata tienen un riesgo aumentado de fractura ósea:

- por hueso frágil por las metástasis óseas

- por la desmineralización en la deprivación androgénica

Los eventos óseos provocan grave alteración de la calidad de vida en este grupo de pacientes, por lo que hay que mejorar el entorno local óseo y evitar la implantación metastásica y retrasar las facturas y reducir el dolor óseo.

La eficacia del Ac. Zoledrónico reduce los eventos óseos relacionados con el hueso en cáncer de próstata metastásico y es útil en la prevención y tratamiento de la osteoporosis secundaria a la supresión androgéncia.

Se sugiere realizar densitometría ósea antes del comienzo del bloqueo androgénico. Se recomienda administrar $4 \mathrm{mg}$ cada 3 meses a los pacientes con metástasis óseas.

\section{Hormonoindependencia}

Es importante definir cual es el momento en que consideramos al paciente hormonoindependiente:

- elevación en dos determinaciones seguidas de PSA

- cuando hay evidencia objetiva de progresión tumoral en los últimos 6 meses

- aumento de PSA post supresión de antiandrógenos

- testosterona plasmática a nivel de castración

En tales circunstancias comienzan a aparecer los primeros trabajos con datos alentadores sobre el rol de la quimioterapia en el cáncer de próstata.

Este tratamiento no debe ser recomendado de forma generalizada. Sólo debería ser indicada en pacientes muy concretos ya que la supervivencia es escasa y la toxicidad significativa.

Además existen evidencias suficientes (ensayos clínicos controlados, apoyados por la Guía Europea en Cáncer de Próstata) para indicar la utilización del ácido Zoledrónico a dosis de 4 $\mathrm{mg} / \mathrm{IV}$ mensualmente para prevenir las complicaciones óseas asociadas a la enfermedad metastásica en estos pacientes. 